\title{
Therapeutic application of purse-string sutures with nylon loops and metal clips under single-channel endoscopy for repair of gastrointestinal wall defects
}

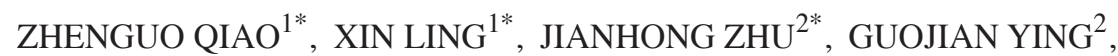 \\ LIHUA XU ${ }^{1}$, HONG ZHU ${ }^{1}$ and JINHAI TANG ${ }^{1}$ \\ ${ }^{1}$ Department of Gastroenterology, The First People's Hospital of Wujiang District, Suzhou, Jiangsu 215200; \\ ${ }^{2}$ Department of Gastroenterology, The Second Affiliated Hospital of Soochow University, Suzhou, Jiangsu 215004, P.R. China
}

Received August 23, 2017; Accepted January 26, 2018

DOI: $10.3892 /$ etm.2018.5956

\begin{abstract}
The present study was performed to evaluate the therapeutic safety and feasibility of purse-string sutures with nylon loops and metal clips under single-channel endoscopy to repair gastrointestinal wall defects that had previously developed during endoscopic full-thickness resection (EFR). A multicenter prospective cohort study of 42 patients who had developed defects of the gastrointestinal wall during EFR was conducted from April 2012 to October 2016. All lesions were endoscopically repaired with either a single-channel gastroscope (research group, $\mathrm{n}=18$ ) or double-channel gastroscope (control group, $n=24$ ). The patients' clinical features, purse-string suturing times and complication rates were analyzed. There was no significant difference in the perforation rate between the research and control groups. There were also no significant differences in the purse-string suturing time (research vs. control group, 10.5 vs. $14.6 \mathrm{~min}$, respectively; $\mathrm{P}=0.214$ ), specimen size or complication rate (subcutaneous emphysema) between the two groups. No recurrences were observed during the follow-up period. The current data suggest that application of purse-string sutures with nylon loops and metal clips for repair of EFR-induced gastrointestinal wall defects may be safely and feasibly applied under single-channel gastroscopy as well as under double-channel gastroscopy.
\end{abstract}

Correspondence to: Dr Jinhai Tang, Department of Gastroenterology, The First People's Hospital of Wujiang District, 169 Gongyuan Road, Suzhou, Jiangsu 215200, P.R. China

E-mail: rujiahuan@163.com

*Contributed equally

Key words: single-channel gastroscope, metal clip, nylon loop, endoscopic submucosal dissection, endoscopic full-thickness resection

\section{Introduction}

With the development of gastrointestinal endoscopy, particularly endoscopic ultrasonography (1), the detection rate of benign submucosal tumors of the digestive tract has gradually increased (2). Submucosal tumors are often considered to be relatively benign; however, they have malignant potential, particularly if they originate from the muscularispropria layer (3). Gastrointestinal stromal tumor (GIST), the most common neoplasm that originates from the muscularispropria layer of the gastrointestinal tract, is diagnosed as malignant in $10-30 \%$ of cases (4). Additionally, the fear of such tumors often causes psychological problems for patients and a subsequent medical burden (5).

Endoscopic full-thickness resection (EFR) (6) is a novel nonsurgical method for radical treatment of submucosal tumors. The application of purse-string sutures with nylon loops and metal clips using a double-channel gastroscope is a commonly used treatment method for defects of the digestive tract resulting from EFR of submucosal tumors $(7,8)$. However, a double-channel gastroscope is not as commonly used as a single-channel gastroscope in the majority of endoscopic centers in China. Thus, the present study aimed to investigate the therapeutic safety and feasibility of the application of purse-string sutures with nylon loops and metal clips under single-channel endoscopy in patients with EFR-induced gastrointestinal wall defects.

A prospective cohort study of 42 patients with gastrointestinal wall defects after EFR was conducted in the First People's Hospital of Wujiang District and the Second Affiliated Hospital of Soochow University (Jiangsu, China). In the present study, the feasibility and safety of purse-string suture placement under single- vs. double-channel gastroscopy was assessed.

\section{Materials and methods}

Medical ethics. The Medical Ethics Committees of The First People's Hospital of Wujiang District (Suzhou, China) and The Second Affiliated Hospital of Soochow University (Suzhou, China) approved the current study. The inclusion criterion was 
the presence of a digestive tract defect following EFR of a submucosal tumor. The exclusion criteria (6) for patients were: i) Non-correctable coagulopathy; ii) severe organ failure; iii) a comorbidity requiring continuous antithrombotic medication; iv) procedure time $>180 \mathrm{~min}$.

Patients. From April 2012 to October 2016 in the endoscopic centers of the First People's Hospital of Wujiang District and the Second Affiliated Hospital of Soochow University, a total of 42 patients (age, $49.0 \pm 16.6$ years; 21 men, $48.3 \pm 18.1$ years; 21 women, $49.5 \pm 16.5$ years) with full-thickness defects of the gastrointestinal wall that had occurred during EFR of submucosal tumors ( 27 in the stomach, 5 in the duodenal bulb and 10 in the rectum) were prospectively investigated. All study participants provided their written informed consent. Two groups were formed using opaque sealed envelopes according to a computer-generated randomized set of numbers. Eighteen patients underwent defect repair using purse-string suture placement with nylon loops and metal clips under a single-channel endoscope (research group), and 24 patients underwent purse-string suture placement with nylon loops and metal clips under a double-channel endoscope (control group).

Medical instruments. The following medical instruments were used during treatment: UM-2R (12 MHZ) and UM-3R (20 MHz) miniature ultrasonic probes (Olympus, Tokyo, Japan), EUM 2000 endoscopic ultrasonography system (Olympus), GIF-260J with flushing function (Olympus), GIF-2T260J with double channel (Olympus), ND-201-11802 cap (Olympus), ITknife2 Electrosurgical Knife (KD-611L; Olympus), KD-620LR hook knife (Olympus), NM-4i-1 injection needle (Olympus), FD-410I hot biopsy forceps (Olympus), MAJ-254 and MAJ-340 nylon loops (Olympus), SD-210U-25 snare (Olympus), HX-600-135 hemostatic clips (Olympus), VIO 200S electrosurgical unit (Erbe Electromedizin $\mathrm{GmbH}$, Tübingen, Germany) and $\mathrm{CO}_{2}$ supply system (Olympus). All procedures were primarily completed by three chief physicians who had performed more than 100 cases of endoscopic submucosal dissection (ESD).

Suture methodfor digestive tract defects. A new suture method was applied in the research group. First, a nylon loop was fixed on a pusher, and the nylon loop was then loosened and tied tightly around the front end of the cap while still holding the handle of the pusher (Fig. 1). Second, the chief endoscopist inserted the endoscope with the nylon loop until the digestive tract defect was reached, and a physician assistant loosened the nylon loop while still holding the handle of the pusher. Third, the chief endoscopist slowly pulled the endoscope backward while the assistant pushed the nylon loop forward until the field of vision was wholly exposed. Fourth, the angle of the metal clips was adjusted to enable the clips to bring the nylon loop to the distal end of the wall defect, and the nylon loop was clipped as tightly as possible into the full layer or muscle layer of the defected wall. Fifth, clipping of the nylon loop to the other side of the defected wall with metal clips was continued using a total of four to six pieces. Sixth, the assistant tightened the handle of the nylon loop to narrow the nylon loop, closed the defected wall, and pulled out the nylon loop pusher.
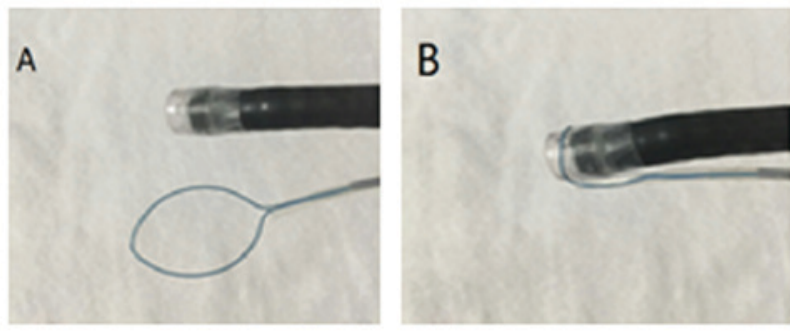

Figure 1. Nylon loop Installation method with single-channel endoscopy. (A) A nylon loop was fixed on a pusher and single-channel gastroscope. (B) The nylon loop was loosened and then tied tightly around the front end of the cap while still holding the handle of the pusher.

Finally, the residual defected wall was clipped with metal clips if necessary (Fig. 2).

The standard suture method was applied in the control group. First, the nylon loop and metal clips were inserted through the two channels of the double-channel endoscope, respectively. Second, the nylon loop was opened under direct endoscopic vision, and the loop was then clipped and fixed to the peripheral edge of the defected wall with metal clips, as in the research group. Third, the assistant tightened the handle of the nylon loop to narrow the nylon loop, closed the defected wall, and pulled out the nylon loop pusher. Finally, the residual defected wall was clipped with metal clips if necessary.

Postoperative management. Fasting, fluid infusion, nutritional support, hemostasis and antibiotics were administered as routine treatment. Nasogastric negative pressure drainage and a semi-reclining position were adopted following surgery when the resected submucosal tumor was located in the upper gastrointestinal tract, and proton pump inhibitors were also administered. Patients were monitored for clinical symptoms and signs, including abdominal pain, abdominal distension, fever, melena, hematemesis and signs of peritonitis. Endoscopy was repeated and the mucosal healing condition was carefully observed 3 months postoperatively. At 6 and 12 months postoperatively, endoscopy was repeated to determine whether any recurrence of the submucosal tumor had occurred.

End points and subgroup analyses. The primary outcome was the success rate of all patients that underwent purse-string suture placement with nylon loops and metal clips, and whether all tumors were resected and taken out. The secondary outcomes were total procedure time and treatment outcomes of ESD (decrease in hemoglobin, days of antibiotic used and average hospital stay). These endpoints were also compared between the two groups.

Statistical analysis. Statistical evaluations were performed using SPSS 13.0 (SPSS Inc., Chicago, IL, USA). Numerical data are expressed as the mean \pm standard deviation, and categorical variables are expressed as mean (percentage). Comparisons between the control group and research group were performed using one-way analysis of variance for continuous variables. Student's t-test was conducted for the continuous variable age. The Pearson's chi-square test was used to test for differences in categorical variables. $\mathrm{P}<0.05$ was considered to indicate a statistically significant difference. 

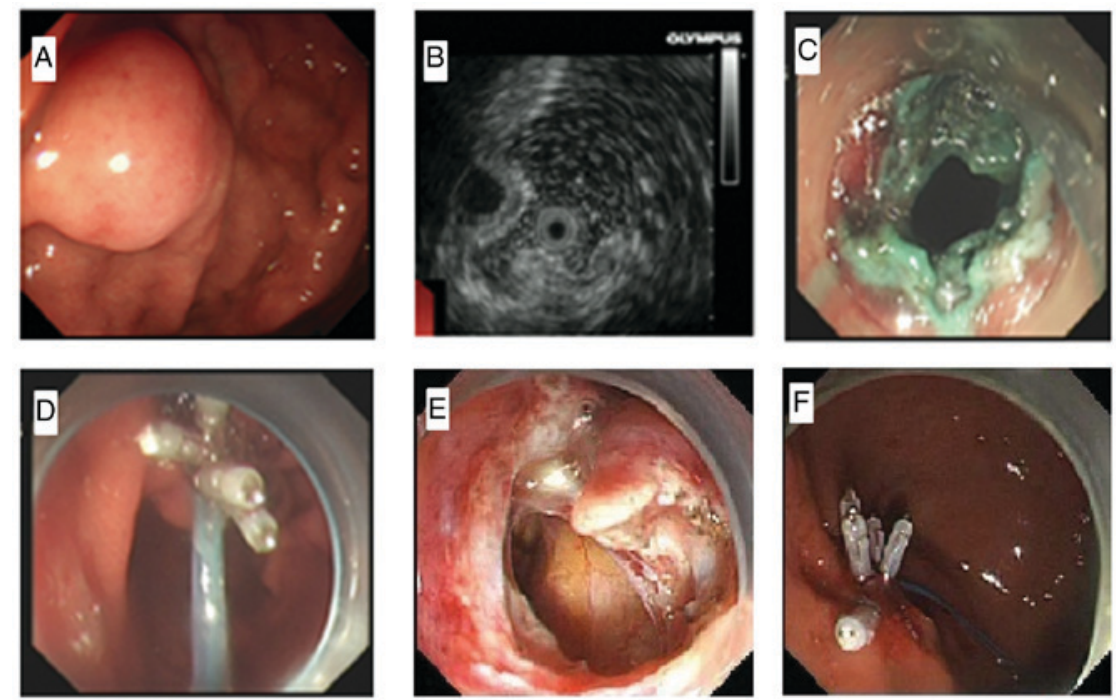

Figure 2. Endoscopic findings prior to and post treatment in (A-D) the research and (E and F) control groups. (A) Submucosal tumors under endoscopy in the antral region of the stomach prior to treatment. (B) The mass was located at the muscularispropria layer by endoscopic ultrasonography. (C) Defect of the digestive tract following endoscopic full-thickness resection. (D) Purse-string suture with a nylon loop and metal clips were used with single-channel endoscopy. (E) Defect of the digestive tract following endoscopic full-thickness resection, the yellow peritoneum was visible. (F) Purse-string suture with a nylon loop and metal clips were used with double-channel endoscopy.

\section{Results}

Patient outcomes. The clinical characteristics of patients are presented in Table I. No significant differences were identified between the two groups $(\mathrm{P}>0.05)$. All 42 patients underwent purse-string suture placement with nylon loops and metal clips with a $100 \%$ success rate. All tumors $(1.0-3.0 \mathrm{~cm})$ were successfully resected and taken out. The procedure time was $10.5 \pm 5.6 \mathrm{~min}$ in the research group and $14.6 \pm 4.5 \mathrm{~min}$ in the control group, with no significant difference $(\mathrm{P}>0.05)$. The mean hospital stay was $4.5 \pm 1.0$ days in the research group and $4.5 \pm 2.5$ days in the control group, with no significant difference $(\mathrm{P}>0.05)$ and no further consultation with a general surgeon or transfer to the general surgery department was required in any patient. The detailed results are presented in Table II.

No cases of hydropneumothorax, mediastinal emphysema or subcutaneous emphysema occurred during the procedure. Eight patients in the research group and 10 patients in the control group underwent abdominal puncture and air drainage due to visible pneumoperitoneum, with no significant difference between the groups $(\mathrm{P}>0.05)$. No postoperative bleeding (hematemesis, melena) was observed in either group.

Postoperative follow-up. Postoperative follow-up was performed for all 42 patients (100\%). The gastrointestinal wall defects had completely healed with no residual metal clips or nylon loops 3 months after EFR treatment. No submucosal tumor recurrences were observed at 6 and 12 months postoperatively.

\section{Discussion}

Endoscopic mucosal resection (EMR) and ESD techniques are now performed worldwide (9). Complications of these procedures include iatrogenic active perforation or unpredictable iatrogenic perforation (10). There is an urgent requirement for endoscopists to repair these gastrointestinal perforations under EMR/ESD/EFR rather than transfer these patients to undergo a general operation. Metal clips are used to close the defects, particularly following ESD and EFR (11). However, when defects of $\geq 3 \mathrm{~cm}$ or severe mucosal edema are present, closure using metal clips alone is challenging (12). Recent developments of the Over-The-Scope Clip system (Ovesco Endoscopy AG, Tübingen, Germany) (13), cutting and sewing machines (14) and artificial repair material (15) have facilitated suturing of iatrogenic perforations following EMR/ESD and EFR; however, the costs of these medical devices and materials are high.

In 2012, Zhong et al (7), successfully closed EMR-induced mucosal defects using a new technique involving purse-string suture placement with metal clips and nylon loops. However, the purse-string sutures were usually placed with a double-channel endoscope in this technique, which limits its widespread clinical use since many hospitals do not have double-channel endoscopes.

Since April 2012 in the First People's Hospital of Wujiang District and the Second Affiliated Hospital of Soochow University the application of purse-string sutures with nylon loops and metal clips under single-channel endoscopy has been successfully used to close EFR-induced gastrointestinal defects in 18 patients. Many other techniques have also been applied since April 2012; for example, the gastric tube insertion method was initially attempted using a single-channel endoscope. This maneuver was performed by insertion of a nylon loop into the gastric cavity first, followed by insertion of the endoscope into the stomach, opening of the nylon loop under direct endoscopic vision, and placement of the nylon loop onto the defects with the help of metal clips. However, because of the hardness of the head of the nylon loop pusher, rough insertion of the nylon loop pusher through the patient's throat could easily cause injury. To avoid this complication, 
Table I. Comparison of clinical characteristics between the two groups.

\begin{tabular}{|c|c|c|c|}
\hline Characteristic & Research group & Control group & P-value \\
\hline No. of patients & 18 & 24 & - \\
\hline Sex (male:female) & $8: 10$ & $13: 11$ & 0.38 \\
\hline Age, years (mean \pm SD) & $48.5 \pm 18.2$ & $49.5 \pm 16.8$ & 0.27 \\
\hline Location of the lesion (S:D:R) & $12: 2: 5$ & $15: 3: 5$ & 0.52 \\
\hline Median maximum tumor diameter, mm (range) & $23.4(17.0-30.0)$ & $23.7(17.0-30.0)$ & 0.34 \\
\hline Median maximum specimen diameter, mm (range) & $29.2(17.0-30.0)$ & $30.6(17.0-30.0)$ & 0.32 \\
\hline
\end{tabular}

SD, standard deviation; S, stomach; D, bulb of duodenum; R, rectum.

Table II. Comparison of outcome measures between the two groups.

\begin{tabular}{|c|c|c|c|}
\hline Outcome & Research group $(n=18)$ & Control group $(n=24)$ & P-value \\
\hline Successful repair, n (\%) & $18(100)$ & $24(100)$ & - \\
\hline Decrease in $\mathrm{Hb}, \mathrm{g} / \mathrm{dl}$, mean $\pm \mathrm{SD}$ & $1.4 \pm 0.7$ & $1.5 \pm 0.8$ & 0.177 \\
\hline Procedure time, min, mean \pm SD & $10.5 \pm 5.6$ & $14.6 \pm 4.5$ & 0.214 \\
\hline Muscle injury, n (\%) & $4(22.2)$ & $6(25.0)$ & 0.146 \\
\hline Postoperative bleeding (hematemesis, melena), n (\%) & $0(0)$ & $0(0)$ & - \\
\hline Postoperative fever, n (\%) & $0(0)$ & $0(0)$ & - \\
\hline Postoperative abdominal pain, n (\%) & $10(55.6)$ & $14(58.3)$ & 0.226 \\
\hline Postoperative sepsis, n (\%) & 0 & 0 & - \\
\hline Postoperative GI tract leakage, n (\%) & 0 & 0 & - \\
\hline Antibiotic use, days, mean \pm SD & $1.5 \pm 0.5$ & $1.5 \pm 0.5$ & 0.245 \\
\hline $\begin{array}{l}\text { Hydropneumothorax/mediastinal } \\
\text { emphysema/subcutaneous emphysema, n (\%) }\end{array}$ & $0 / 0 / 0(0 / 0 / 0)$ & $0 / 0 / 0(0 / 0 / 0)$ & - \\
\hline Pneumoperitoneum, n (\%) & $8(44.4)$ & $10(41.7)$ & 0.189 \\
\hline Restart food on POD 3, n (\%) & $14(77.8)$ & $18(75.0)$ & 0.381 \\
\hline Hospital stay, days, mean \pm SD & $4.5 \pm 1.0$ & $4.5 \pm 2.5$ & 0.600 \\
\hline
\end{tabular}

$\mathrm{Hb}$, hemoglobin; SD, standard deviation; GI, gastrointestinal; POD, postoperative day.

a new technique was developed. This involved synchronous insertion of the nylon loop pusher along with the endoscope, temporary fixation of the nylon loop onto the head of the cap, insertion of the endoscope with the nylon loop to the gastrointestinal wall defects, loosening of the nylon loop and removal of the cap, and gradual purse-string suture placement after metal clip insertion through the channel of the endoscope.

Certain advantages of single-over double-channel endoscopy were also identified by practicing this maneuver using a single-channel endoscope in 18 patients. First, when the nylon loop is inserted into the gastrointestinal tract using a single-channel endoscope, placement of the loop onto the defect under the guidance of metal clips is considerably easier than with a double-channel endoscope, with which the direction of the clips and nylon loops is relatively fixed. Second, reversal of the endoscope to complete the suture placement is more difficult with a double- than single-channel endoscope. Third, better operational space and flexibility are achieved when the endoscopists and assistants independently reach the defect with the endoscope and nylon loop under single-channel endoscopy.
Collaboration between the endoscopist and assistant is essential to complete the purse-string suture placement.

In the current prospective study, no significant differences in operation time, occurrence of bleeding, occurrence of fever, rate of 3-day postoperative resumption of an oral diet or hospital stay were identified between the research and control groups. This lack of differences suggests that purse-string suture placement under single-channel endoscopy is as safe and efficient as under double-channel endoscopy.

In conclusion, purse-string suture placement with nylon loops and metal clips under single-channel endoscopy appears to be as safe, economical, convenient and efficient as that under double-channel endoscopy. Furthermore, because endoscopists and assistants manipulate the nylon loop pusher and metal clips independently under a single-channel endoscope, a single-channel scope has better operational space and flexibility compared with a double-channel endoscope, making full-thickness suturing of digestive tract defects easier to perform. It is worth considering the widespread application of single-channel endoscopes for repair of such defects, particularly in hospitals without a double-channel endoscope. 


\section{Acknowledgements}

The authors would like to thank Dr Angela Morben for editing the English text of an earlier version of this manuscript.

\section{Funding}

This study was supported by the Program for the Talents in Science and Education of Jiangsu Province, China (grant no. Z2017012) and Program for the Talents in Science and Education of Wujiang District, Suzhou, China (grant no. WWK201517).

\section{Availability of data and materials}

The datasets used and/or analyzed during the present study are available from the corresponding author on reasonable request.

\section{Authors' contributions}

ZQ, XL and JZ performed the endoscopy procedure, designed the study and drafted the manuscript. GY conceiving the study, designed the study and collected the data. LX collected the data, identified the picture of the endoscopy, coordinated with the hospital administration and obtained consent from the patients. HZ performed statistical analysis, interpreted the data and followed up with the patients. JT performed the endoscopy procedures and was responsible for the conception and design of the study. All authors read and approved the final manuscript.

\section{Ethics approval and consent to participate}

This study was approved by the Ethics Committee of the First People's Hospital of Wujiang District and the Second Affiliated Hospital of Soochow University.

\section{Consent for publication}

Patients provided written informed consent for the publication of their data.

\section{Conflict of interest}

The authors declare that they have no competing interests.
2. Rösch T, Lorenz R, Dancygier H, von Wickert A and Classen M: Endosonographic diagnosis of submucosal upper gastrointestinal tract tumors. Scand J Gastroenterol 27: 1-8, 1992.

3. Chun SY, Kim KO, Park DS, Lee IJ, Park JW, Moon SH, Baek IH, Kim JH, Park CK and Kwon MJ: Endoscopic submucosal dissection as a treatment for gastric subepithelial tumors that originate from the muscularis propria layer: A preliminary analysis of appropriate indications. Surg Endosc 27: 3271-3279, 2013.

4. Mandrioli M, Mastrangelo L, Masetti M, Zanini N, Lega S, Nannini M, Gruppioni E, Altimari A, Dei Tos AP, Fabbri C and Jovine E: Characterization of malignant gastrointestinal stromal tumors-a single center experience. J Gastrointest Oncol 8: 1037-1045, 2017.

5. Berzin TM, Blanco PG, Lamont JT and Sawhney MS: Persistent psychological or physical symptoms following endoscopic procedures: An unrecognized post-endoscopy adverse event. Dig Dis Sci 55: 2869-2873, 2010.

6. Li QL and Zhou PH: Perspective on peroral endoscopic myotomy for achalasia: Zhongshan experience. Gut Liver 9: 152-158, 2015.

7. Zhong YS, Shi Q, Yao LQ, Zhou PH, Xu MD, Ma LL and Chen T: Complete closure of gastric wall defect after endoscopic full-thick resection with metal clips and endoloop snare. Zhonghua Wei Chang Wai Ke Za Zhi 15: 280-284, 2012 (In Chinese).

8. Shi Q, Chen T, Zhong YS, Zhou PH, Ren Z, Xu MD and Yao LQ. Complete closure of large gastric defects after endoscopic full-thickness resection, using endoloop and metallic clip interrupted suture. Endoscopy 45: 329-334, 2013.

9. Sun W, Wu S, Han X and Yang C: Effectiveness of endoscopic treatment for gastrointestinal neuroendocrine tumors: A retrospective study. Medicine (Baltimore) 95: e3308, 2016.

10. Kim GJ, Park SM, Kim JS, Ji JS, Kim BW and Choi H: Risk factors for additional surgery after iatrogenic perforations due to endoscopic submucosal dissection. Gastroenterol Res Pract 2017: 6353456, 2017.

11. Li QL, Yao LQ, Zhou PH, Xu MD, Chen SY, Zhong YS, Zhang YQ, Chen WF, Ma LL and Qin WZ: Submucosal tumors of the esophagogastric junction originating from the muscularis propria layer: A large study of endoscopic submucosal dissection (with video). Gastrointest Endosc 75: 1153-1158, 2012.

12. Fujishiro M, Yahagi N, Kakushima N, Kodashima S, Muraki Y, Ono S, Kobayashi K, Hashimoto T, Yamamichi N, Tateishi A, et al: Successful nonsurgical management of perforation complicating endoscopic submucosal dissection of gastrointestinal epithelial neoplasms. Endoscopy 38: 1001-1006, 2006.

13. Weiland T, Fehlker M, Gottwald T and Schurr MO: Performance of the OTSC system in the endoscopic closure of iatrogenic gastrointestinal perforations: A systematic review. Surg Endosc 27: 2258-2274, 2013.

14. Li Y, Wu JH, Meng Y, Zhang Q, Gong W and Liu SD: New devices and techniques for endoscopic closure of gastrointestinal perforations. World J Gastroenterol 22: 7453-7462, 2016.

15. Cios TJ, Reavis KM, Renton DR, Hazey JW, Mikami DJ, Narula VK, Allemang MT, Davis SS and Melvin WS: Gastrotomy closure using bioabsorbable plugs in a canine model. Surg Endosc 22: 961-966, 2008.

This work is licensed under a Creative Commons Attribution-NonCommercial-NoDerivatives 4.0 International (CC BY-NC-ND 4.0) License.

\section{References}

1. Caletti G, Fusaroli P and Bocus P: Endoscopic ultrasonography. Digestion 59: 509-529, 1998. 